\title{
Restrição de crescimento seletiva em gestação gemelar monocriônica: relato de caso
}

\section{Selective growth restriction in monochorionic twin pregnancy: a case report}

Pedro Wilson Leitão Lima Filho ${ }^{1}$. Francisco Edson de Lucena Feitosa ${ }^{1}$. Helvécio Neves Feitosa ${ }^{1,2}$. Raimundo Homero de Carvalho Neto ${ }^{1}$. Francisco Herlânio Costa Carvalho ${ }^{1}$.

1 Maternidade-Escola Assis Chateaubriand, Universidade Federal do Ceará (UFC). 2 Universidade de Fortaleza (UNIFOR).

RESUMO

A restrição de crescimento fetal (RCF) ocorre em aproximadamente 3 a $10 \%$ das gestações únicas, em 9,1\% de todos os gêmeos, e em $9,9 \%$ dos gêmeos monocoriônicos. O déficit de crescimento pode comprometer apenas um dos gêmeos de forma seletiva (RCF-S). A morte espontânea do gêmeo com restrição pode resultar em morte concomitante ou deficiência neurológica grave do outro gêmeo.

Há classificação em três subtipos para essa entidade clínica, onde os de tipo I (Doppler normal) são conduzidos de forma expectante com avaliação periódica ao Doppler. Para os subtipos II (Doppler anormal mantido) e os subtipos III (Doppler anormal intermitente) não há consenso na literatura, com tendência a realização do laser para separação das circulações com o intuito de evitar as conseqüências adversas decorrentes da morte potencial do gêmeo com RCFs.

O objetivo deste relato de caso é apresentar uma evolução de RCFs Tipo I conduzida de forma conservadora com resultado satisfatório e discutir os aspectos desta importante entidade na obstetrícia.

Palavras-chave: Retardo do crescimento fetal. Gravidez de gêmeos. Ultrassonografia pré-natal. Monitorização fetal. Conduta expectante.

\section{ABSTRACT}

The fetal growth retardation (FGR) occurs in approximately 3-10\% of singleton pregnancies, $9.1 \%$ of all twins, and in $9.9 \%$ of monochorionic twins. The growth deficit can compromise only one twin selectively (FGR-S). Spontaneous twin death restriction can result in death or serious neurological concomitant deficiency of other twin.

There are classified into three subtypes for this clinical entity, where the type I (normal Doppler) are conducted in an expectant way with periodic evaluation by Doppler echocardiography. For subtypes II (abnormal Doppler maintained) and subtypes III (intermittent abnormal Doppler) there is no consensus in the literature, with a tendency to completion of the laser to separate the circulations in order to avoid the adverse consequences of the potential death of twin with RCFs .

The objective of this report is to present a progress RCFs Type I conducted conservatively with satisfactory results and discussing this important entity in obstetrics.

Keywords: Fetal growth retardation. Twin pregnancy. Prenatal ultrasound. Fetal monitoring. Expectant management.

Autor correspondente: Pedro Wilson Leitão Lima Filho. E-mail: pedrowf@yahoo.com.br.

Conflito de interesses: Não há qualquer conflito de interesses por parte de qualquer um dos autores.

Recebido em: 02 Mai 2015; Revisado em: 08 Jun 2015; Aceito em: 19 Jun 2015. 


\section{INTRODUÇÃO}

A restrição de crescimento fetal seletiva (RCF-S) em gêmeos monocoriônicos está associada a um aumento substancial da morbidade e mortalidade perinatal para ambos os gêmeos. A evolução clínica depende da combinação dos efeitos da insuficiência placentária no gêmeo com RCF com as transferências de sangue entre os gêmeos através de anastomoses placentárias. ${ }^{1-3}$

As RCF-S podem ser classificadas de acordo com as características do fluxo diastólico na artéria umbilical no gêmeo com RCF; permitindo assim a diferenciação de grupos clínicos com prognósticos diferentes. O tipo I de RCFs tem fluxo diastólico normal e é de relativamente bom prognóstico. O tipo II é definido pelo fluxo diastólico final persistentemente ausente/reverso e está associada a um alto risco de morte intra-uterina do gêmeo com RCF e/ou parto muito prematuro. O tipo III é definido pela presença de fluxo diastólico final ausente/reversa intermitente, e está associada ao risco de 10 a $20 \%$ de morte fetal inesperada do menor gêmeo e 10 a $20 \%$ de lesão neurológica no gêmeo maior. ${ }^{1,4}$

A estratégia no manejo de RCF-S com Doppler anormal da artéria umbilical (tipos II e III) continua a ser um desafio, e pode incluir terapia fetal eletiva ou estreita vigilância com a terapia fetal ou parto eletivo na presença de deterioração fetal grave. $^{1,2}$

As conseqüências adversas decorrentes da morte potencial do gêmeo com RCF-S podem ser evitadas desvinculando a circulação entre os dois fetos. Isso pode ser feito desfazendo as anastomoses placentárias através da fotocoagulação a laser de vasos comunicantes ou, em casos extremos, através da realização da oclusão do cordão umbilical quando o prognóstico para o feto menor já é muito reservado. ${ }^{1,2}$

Provavelmente não há melhor ou única estratégia, uma vez que as decisões serão finalmente influenciadas pela gravidade da RCF, idade gestacional, a vontade dos pais e questões técnicas.

\section{RELATO DE CASO}

Paciente MFFF, 42 anos, G2P1A0, casada, católica, natural e procedente do interior do Ceará. Realizou inseminação artificial em maio de 2014, com transferência de dois embriões, tendo como resultado a fertilização de um deles que posteriormente se dividiu, havendo assim a formação de uma gestação gemelar monocoriônica. Sem outras comorbidades. Relatava ultrassom morfológico de primeiro trimestre, realizado na $13^{\mathrm{a}}$. semana de gestação, com discordância das translucências nucais (TN) e das biometrias fetais. O comprimento cabeça-nádega do feto 1 foi de $71 \mathrm{~mm}$ e do feto 2 de $64 \mathrm{~mm}$ (diferença de 9,9\%). As TNs do 1,6 $\mathrm{mm}$ e a de $3,4 \mathrm{~mm}$, respectivamente. Ossos nasais presentes em ambos os fetos. Doppler de ducto venoso com padrão anormal (onda "A" com fluxo reverso) em ambos. Os riscos para anomalias cromossômicas calculados pelo software versão 2.8 da Fetal Medicine Foundation (UK registered charity 1037116) ${ }^{\circledR}$ foram: 1:4 para trissomia do cromossomo 21, 1:10 para trissomia do 18 e 1:71 para trissomia do 13 .
Ofereceu-se, então, realização de amniocentese para cariótipos fetais. A família decidiu por não realizar a investigação fetal invasiva. Ficou em acompanhamento seriado pelo risco aumentado para síndrome de transfusão feto-fetal (STFF). Foi encaminhada para o Serviço de Medicina Materno Fetal da Maternidade-Escola Assis Chateaubriand (UFC) com idade gestacional de 19 semanas e 5 dias para investigação de suposta STFF e melhor seguimento.

Avaliação detalhada no serviço afastou o diagnóstico de síndrome de transfusão feto-fetal, pois as quantidades de líquido amniótico de ambas as bolsas mantiveram-se sempre normais e as bexigas de ambos os fetos sempre visibilizadas aos ultrassons de seguimento. $\mathrm{O}$ peso do feto 2 encontrava-se abaixo do percentil 10 para qualquer curva de normalidade (gestações únicas ou duplas) configurando o diagnóstico de Restrição de Crescimento Fetal seletiva em gestação monocoriônica. Doppler de artéria umbilical de ambos normais - classificação de Gratacós tipo I. ${ }^{4}$ Decidiu-se pelo seguimento ultrassonográfico periódico (inicialmente semanal e posteriormente a cada duas semanas). O perfil hemodinâmico de ambos os fetos mantiveram-se normais durante todo o acompanhamento com progressão das diferenças entre os pesos fetais (Quadro 1).

Quadro 1. Resumo da evolução ultrassonográfica da gestação gemelar monocoriônica-diamniótica com RCF-S.

$\begin{array}{lllll}\begin{array}{l}\text { Idade } \\ \text { Gestacional }\end{array} & \begin{array}{l}\text { Peso } \\ \text { Feto 1 }\end{array} & \begin{array}{l}\text { Peso } \\ \text { Feto 2 }\end{array} & \begin{array}{l}\text { Diferença } \\ \text { entre os } \\ \text { pesos } \\ \text { fetais }\end{array} & \begin{array}{l}\text { Doppler-Perfis } \\ \text { hemodinâmicos }\end{array} \\ & & & & \end{array}$

$\begin{array}{lcccc}21 \mathrm{~s} & 421 \mathrm{~g} & 309 \mathrm{~g} & 27 \% & \text { Normais } \\ 23 \mathrm{~s} 5 \mathrm{~d} & 607 \mathrm{~g} & 447 \mathrm{~g} & 26 \% & \text { Normais } \\ 28 \mathrm{~s} & 1272 \mathrm{~g} & 748 \mathrm{~g} & 41 \% & \text { Normais } \\ 30 \mathrm{~s} & 1489 \mathrm{~g} & 869 \mathrm{~g} & 42 \% & \text { Normais }\end{array}$

Paciente foi submetida a parto cesárea no dia 23 de dezembro de 2014, com idade gestacional de 34 semanas e 5 dias. O peso do feto 1 foi 1919 gramas, Apgar 9/9 e recebeu alta no mesmo dia. o peso do feto 2 foi de 1230 gramas, Apgar 9/9 e permaneceu internado por 20 dias em ar ambiente, sem complicações, para ganhar peso. A diferença entre os fetos fetais ao nascimento foi de $36 \%$.

\section{DISCUSSÃO}

A definição de RCF no período gestacional se confunde com a de pequeno para idade gestacional que é feita pela estimativa do peso fetal abaixo do percentil 10 de uma curva de normalidade; no entanto, pela objetividade da avaliação é a definição que tem prevalecido na maioria dos serviços, apesar das críticas e da dificuldade no uso de curvas adequadas. Pode acometer um ou ambos os fetos da gestação 
gemelar monocoriônica e diamniótica. A discordância de peso entre os fetos da gestação gemelar muitas vezes também é confundida com o diagnóstico de RCF-S nos casos de discordância de peso $\geq 25 \%$. A discordância é calculada como a diferença entre o peso fetal estimado do maior gêmeo e o menor, dividido pelo peso fetal estimado do maior gêmeo e também apresenta piora nos prognósticos perinatais, mas são entidades diferentes. ${ }^{1,3,5-9}$

A RCF-S ocorre quando um dos fetos de uma gestação gemelar tem padrão de crescimento abaixo do percentil $10 \mathrm{e}$ o cogemelar apresenta adequado padrão de crescimento. Nesses casos, é comum encontrar-se padrão de discordância de peso fetal acima de 25\%. A frequência de RCF-S em gestações monocoriônicas e diamnióticas oscila entre $10 \%$ e $15 \%$. ${ }^{1,3,9}$

Estudos mostram que quase $50 \%$ das gestações monocoriônicas apresentam inserção velamentosa ou marginal de cordão no leito placentário. Nos casos de inserção central e periférica (especialmente velamentosa), o cordão com inserção central assumirá grande parte do parênquima placentário, deixando a porção referente à inserção marginal bastante reduzida. ${ }^{1}$

A placenta em gestações monocoriônicas é basicamente semelhante a uma placenta de únicos, exceto na porção intermediária entre as duas inserções de cordão. Nesta área neutra entre os fetos, há uma competição pelos cotilédones. Desta forma, há cotilédones que são perfundidos pela artéria de um gêmeo (doador), mas a veia correspondente que emerge do mesmo forame é capturada pelo outro gêmeo (receptor), sendo esta a base anatômica das anastomoses arteriovenosas $(\mathrm{A}-\mathrm{V})$. As anastomoses arterioarteriais (A-A) e venovenosas $(\mathrm{V}-\mathrm{V})$, são de estrutura mais simples e estão localizadas na superfície da placa coriônica. ${ }^{1}$

Em gestações monocoriônicas e diamnióticas balanceadas, existem muitas anastomoses $\mathrm{A}-\mathrm{V}$ e elas correrão em diferentes sentidos. Aparentemente, as anastomoses A-A tem um efeito protetor em casos de desbalanceamento das anastomoses A-V. Estas são as principais bases placentárias para as principais complicações da monocorionidade, como a STFF e RCF-S. ${ }^{1}$

A RCF-S é classificada em tipo I, II e III. No tipo I o fluxo diastólico é normal na artéria umbilical do gêmeo pequeno, as anastomoses da placenta em gestações tipo I são semelhantes as das gestações normais. O tipo I tem um bom prognóstico e as taxas de mortalidade intra-uterina são de aproximadamente 2 a $4 \%$. A conduta deve ser expectante e o acompanhamento rigoroso para descartar progressão para o tipo II. $\mathrm{Na}$ ausência de tal progressão, controle ultrassonográfico com Doppler quinzenal ou semanal pode ser feito. Na maioria dos casos, o feto RCF-S permanecerá com um Doppler normal até próximo o termo da gravidez, permitindo a resolução eletiva da gestação entre 34 e 35 semanas. ${ }^{1,3,4}$

O tipo II é caracterizado por fluxo diastólico final persistentemente ausente ou reverse na artéria umbilical. Como no tipo I, o tipo II de RCFS apresenta uma distribuição de anastomoses placentárias bastante semelhante aos gêmeos monocoriônicos não complicados, mas com uma discordância placentária mais grave. $\mathrm{O}$ território fetal do gêmeo com RCF-S geralmente é extremamente pequeno no tipo II, mas a relação peso fetal/discordância placentária é significativamente menor do que em gêmeos monocoriônicos não complicados, demonstrando assim como a transfusão de sangue entre os gêmeos atenua a gravidade da restrição de crescimento. O tipo II apresenta um alto risco de morte intrauterina ou parto prematuro, pois a insuficiência placentária é mais grave no tipo II que no tipo I e não pode ser compensada pela transfusão entre os gêmeos. Devido à deterioração do estado do gêmeo com RCF-S, a resolução da gestação na maioria dos casos no tipo II se dá por volta de 28 a 30 semanas de gestação. ${ }^{1,3,4}$

A conduta dependerá da idade gestacional e do grau de deterioração do feto com RCF-S. Conduta expectante pode ser tomada nos casos em que ocorre uma deterioração antes da viabilidade fetal ser atingida ou através da resolução eletiva da gestação depois de 28 semanas, sendo feita por meio de um acompanhamento semanal quando o Doppler do ducto venoso é normal e um acompanhamento mais frequente quando o índice de pulsatilidade do ducto venoso está elevado acima de dois desvios-padrão. Já a conduta ativa pode ser feita pela oclusão do cordão umbilical, que é um procedimento mais simples, com menor risco e taxa de sobrevida para o gêmeo normal de 80 a $90 \%$ ou por meio da fotocoagulação com laser, que é mais complexa, conduz a morte do feto com RCF-S e menor taxa de sobrevida do feto normal.

O tipo III é definido como fluxo diastólico final intermitente na artéria umbilical. A principal característica desse padrão de Doppler é a alternância de fases com fluxo diastólico positivo e fluxo diastólico ausente ou reverso intermitente, em geral de forma cíclica. ${ }^{1,3,6}$

Na maioria dos casos do tipo III, o efeito compensador do grande número de anastomoses arterio-arteriais permite a sobrevivência do feto com RCF-S até estágios avançados da gravidez, sem mostrar sinais claros de deterioração hipóxica. Nesse aspecto, o comportamento pode assemelhar-se ao do tipo I. No entanto, ao contrário do tipo I, o tipo III está associado a aumento significativo do risco de morte intraútero inesperada do feto com RCF-S e de lesão cerebral no gêmeo normal. Estes efeitos adversos são explicados pelo alto risco de acidentes hemorrágicos agudos de grandes vasos arterio-arteriais, que podem levar à morte do gêmeo menor ou hipovolemia aguda no maior. ${ }^{1,3,6}$

O prognóstico em geral é melhor do que no tipo II, mas a escolha de que conduta tomar é difícil, não só pela imprevisibilidade dos resultados adversos, como também por existir mais de uma opção de tratamento. Se a conduta expectante for escolhida, o acompanhamento deve ser semelhante ao discutido acima para os casos do tipo II, ou seja, acompanhamento semanal se o Doppler venoso estiver normal, e mais frequente sem deixar de considerar conduta ativa se o Doppler venoso estiver alterado. Infelizmente, no tipo III do feto RCF-S raramente irá mostrar sinais de deterioração fetal no Doppler venoso, portanto, uma opção 
razoável seria resolver eletivamente a gestação por volta de 32 semanas. Esta decisão não se baseia em qualquer prova, mas as razões são provavelmente semelhantes às utilizadas para as decisões em gravidezes monoamnióticas, isto é, reduzir a possibilidade de eventos adversos. Se a escolha for a terapia fetal, pode ser realizada tanto a oclusão do cordão umbilical, como a fotocoagulação a laser, da mesma forma dos casos do tipo II, preferencialmente através da oclusão do cordão umbilical, não somente por essa técnica oferecer menos riscos de eventos adversos, mas também por ser de execução mais fácil que a fotocoagulação a laser, que deve ser realizada apenas por cirurgiões experientes. ${ }^{1,3,6-8}$

Diante de casos de gestações monocoriônicas e diamnióticas com suspeita de restrição de crescimento fetal seletivo o primeiro passo é fazer diagnóstico diferencial com síndrome da transfusão feto-fetal que pode apresentar evolução e condutas diferentes. Após confirmação do diagnóstico da RCF-S não há uma conduta única para o acompanhamento e tratamento dessa enfermidade, pois fatores como a gravidade da RCF-S, a idade gestacional, a vontade dos pais e questões técnicas influenciarão na escolha de como deve ser conduzido cada caso. Deve-se sempre estar atentos para evitar ou minimizar os possíveis efeitos adversos que esta condição poderá causar. ${ }^{1,6-9}$

\section{REFERÊNCIAS}

1. Valsky DV, Eixarch E, Martinez JM, Crispi F, Gratacós E. Selective intrauterine growth restriction in monochorionic twins: Pathophysiology, diagnostic approach and management dilemmas. Semin Fetal Neonatal Med. 2010;15(6):342-8.

2. Chalouhi GE, Stirnemann JJ, Salomon LJ, Essaoui M, Quibel T, Ville Y. Specific complications of monochorionic twin pregnancies: twin-twin transfusion syndrome and twin reversed arterial perfusion sequence. Semin Fetal Neonatal Med. 2010;15(6):349-56.

3. Russell Z, Quintero RA, Kontopoulos EV. Intrauterine growth restriction in monochorionic twins. Semin Fetal Neonatal Med. 2007;12(6):439-49.

4. Gratacós E, Lewi L, Muñoz B, Acosta-Rojas R, HernandezAndrade E, Martinez JM, et al. A classification system for selective intrauterine growth restriction in monochorionic pregnancies according to umbilical artery Doppler flow in the smaller twin. Ultrasound Obstet Gynecol. 2007;30(1):28-34.

5. Lopriore E, Slaghekke F, Vandenbussche FP, Middeldorp JM, Walther FJ, Oepkes D. Cerebral injury in monochorionic twins with selective intrauterine growth restriction and/or birth weight discordance. Am J Obstet Gynecol. 2008;199(6):628.e1-5.
O caso relatado apresenta um seguimento adequado de gestação gemelar monocoriônica/diamniótica com achado de RCF-S: primeiro foi realizado ultrassom morfológico de primeiro trimestre com sinais de risco para evolução com STFF ou RCF-S, ou seja, discordância das TNs e das biometrias fetais. O seguimento ultrassonográfico dessas gestações deve ser quinzenal entre 16 e 26 semanas, mesmo que no morfológico de I trimestre não se encontre sinais de maior risco para essas entidades. No caso relatado a presença desses sinais aumentou a vigilância em busca de um diagnóstico mais precoce.

$\mathrm{Na} 19^{\mathrm{a}}$ semana de gestação houve a suspeita, não confirmada, de STFF. Melhor avaliação confirmou RCF-S Tipo I. O seguimento foi apropriado (inicialmente quinzenal e depois semanal até o parto) em serviço especializado de Medicina Fetal. Conforme as avaliações com Doppler se mantiveram normais, ou seja, persistiu na classificação de tipo I a conduta adotada foi conservadora até a idade gestacional de 34 semanas quando a viabilidade dos conceptos no nosso meio é a regra.

6. Gratacós E, Antolin E, Lewi L, Martínez JM, HernandezAndrade E, Acosta-Rojas R, et al. Monochorionic twins with selective intrauterine growth restriction and intermittent absent or reversed end-diastolic flow (type III): feasibility and perinatal outcome of fetoscopic placental laser coagulation. Ultrasound Obstet Gynecol. 2008;31(6):669-75.

7. Chalouhi GE, Marangoni MA, Quibel T, Deloison B, Benzina N, Essaoui $\mathrm{M}$, et al. Active management of selective intrauterine growth restriction with abnormal Doppler in monochorionic diamniotic twin pregnancies diagnosed in the second trimester of pregnancy. Prenat Diagn. 2013;33(2):109-115.

8. Lanna MM, Rustico MA, Dell'Avanzo M, Schena V, Faiola S, Consonni D, et al. Bipolar cord coagulation for selective feticide in complicated monochorionic twin pregnancies: 118 consecutive cases at a single center. Ultrasound Obstet Gynecol. 2012;39(4):407-413.

9. Cosmi E, Visentin S, Favretto D, Tucci M, Ragazzi E, Viel G, et al. Selective intrauterine growth restriction in monochorionic twin pregnancies: markers of endothelial damage and metabolomic profile. Twin Res Hum Genet. 2013;16(4):816-26.

\section{Como citar:}

Lima PW, Feitosa FE, Feitosa HN, Carvalho RH Neto, Carvalho FH. Restrição de crescimento seletiva em gestação gemelar monocriônica: relato de caso. Rev Med UFC. 2015 jan-jun;55(1):57-60. 